\title{
Linking microbial community on grapes from two Portuguese wine regions to the biogenic amines production in musts
}

\author{
Rita Calisto ${ }^{1}$, Ana Patrícia Graça ${ }^{1,2}$, Ana Rita Lopes ${ }^{3}$, Paula Araújo ${ }^{4}$, Eugénia Pinto ${ }^{2,3}$, Joana Oliveira ${ }^{4}$, \\ José A.M. Catita ${ }^{5,6}$, Natacha Fontes ${ }^{7}$, António Graça ${ }^{7}$, and Olga Maria Lage ${ }^{1,2}$ \\ ${ }^{1}$ Department of Biology, Faculty of Sciences, University of Porto, Portugal \\ ${ }^{2}$ Interdisciplinary Centre of Marine and Environmental Research, University of Porto, Portugal \\ ${ }^{3}$ Department of Biological Sciences, Faculty of Pharmacy, University of Porto, Portugal \\ ${ }^{4}$ REQUIMTE- LAQV, Department of Chemistry and Biochemistry, Faculty of Sciences, University of Porto, Portugal \\ ${ }^{5}$ Paralab, SA \\ ${ }^{6}$ CEBIMED - Faculty of Health Sciences; University Fernando Pessoa, Porto, Portugal \\ ${ }^{7}$ Sogrape Vinhos, S.A.
}

\begin{abstract}
Grapevine-associated microbiota influences wine organoleptic properties. Spoilage due to undesired microorganisms and biogenic amines (BAs) presence are two main constrains that must be seriously considered. In wine, BAs can originate from the grape berries or can be produced during fermentation, ageing or storage. This work aimed to understand if the high BAs levels observed in musts can have its origin in the microbial community present on grapes. The following methodologies were done: bacterial and fungal grapes communities' isolation, BAs quantification in grapes and musts and molecular amplification of the genes related to BAs production. For comparative purposes, microbial communities from grapes and musts from Douro (low BAs levels in musts) and Alentejo (high BAs levels in musts) were used. Higher number and diversity of bacteria were observed in Alentejo grapes comparatively to Douro ones. Filamentous fungi were predominant when compared with yeasts and the diversity was higher in Alentejo. BAs levels mainly due to putrescin were about ten times higher in grapes and musts from Alentejo. As bacteria isolated from Alentejo grapes showed a great BAs-production potential, namely putrescin, our results suggest a bacterial grape origin for the high putrescine levels found in fresh musts of this region.
\end{abstract}

\section{Introduction}

The production of wine is an important industry with a major economic and social impact in Portugal and in many other countries. Although microorganisms play a fundamental role on the entire process of wine production and strains responsible for wine fermentation are well known and studied, the grape-bunch associated microbial communities are still poorly characterized and their role in wine processing very much unknown [1-3]. Their influence on wine terroir is gaining relevance [3]. However, the microbial community can also induce noxious effects with great impact on this industry. Spoilage microorganisms can grow during fermentations or the storage period [4]. One problem faced during wine making in specific situations is the appearance of high levels of Biogenic Amines (BAs) which may have a microbial origin. BAs are secondary metabolites of organic nitrogenous nature and putrescine, histamine, tyramine and cadaverine are the main BAs associated to wine [4]. High BA levels in wine can be potentially toxic to humans and their effect can even be potentiated by alcohol [5].

The aim of this work was to assess the microbial communities present on grapes from two Portuguese wine producing regions (Douro and Alentejo) with known differences in the levels of must BAs and correlate these levels to the bacterial potential capacity for BAs production.

\section{Material and methods}

Wine grape sampling in Douro and Alentejo was done one month before (D1 and A1) and at harvesting (D2 and A2). Grapes collected aseptically were transported under cold conditions and processed within 24h. Musts were collected immediately after crushing, under the same conditions in Douro and Alentejo. Isolation was carried out using grape extracts in a non-selective broad medium for heterotrophic bacteria (NA medium $\mathrm{pH} 5.5$ and 7.0: $0.5 \%$ peptone, $0.3 \%$ yeast extract and $1.5 \%$ agar) and in appropriate media for yeasts and filamentous fungi (yeast peptone glucose (YPG) supplemented with biphenyl and chloramphenicol, $\mathrm{pH}$ 5.0; Sabouraud dextrose agar (SAB) supplemented with chloramphenicol, pH 5.6; YPG supplemented with biphenyl, chloramphenicol and cycloheximide (YPGciclo), pH 5.0; SAB supplemented with chloramphenicol and cycloheximide (SABciclo), $\mathrm{pH}$ 6.9). BAs levels were quantified in the juice extracted from sampled grapes and in musts immediately after harvesting [6]. Bacterial isolates were phylogenetically identified based on the analysis of the 16S rRNA gene [7] and screened for the presence of the genes involved in the production of BAs: cadaverine, putrescine, tyramine and 


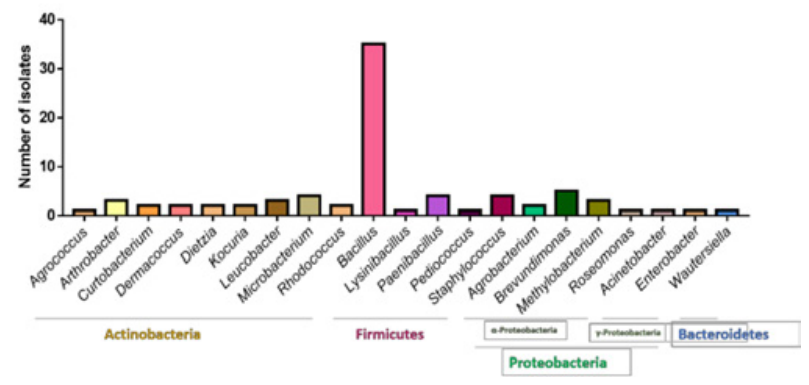

Figure 1. Affiliation of the isolates to corresponding genus and phyla or class. Although the Firmicutes Bacillus was the most abundant genus, Actinobacteria was de most diversified group (9 different genera).

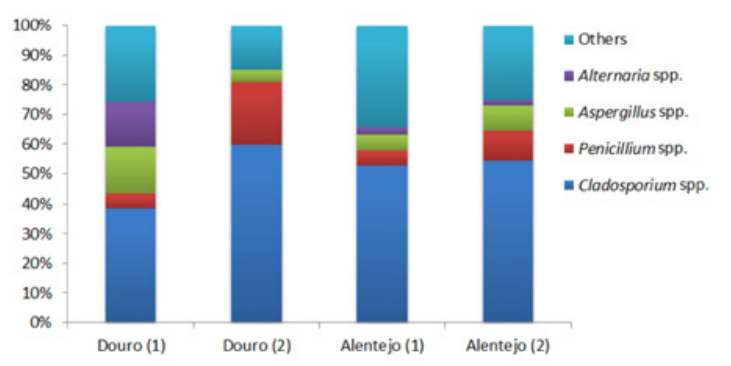

Figure 2. Filamentous fungi isolated from grapes sampled in Douro and Alentejo. Douro (1) - Douro one month before harvesting; Douro (2) - Douro at harvesting; Alentejo (1) Alentejo one month before harvesting; Alentejo (2) - Alentejo at harvesting.

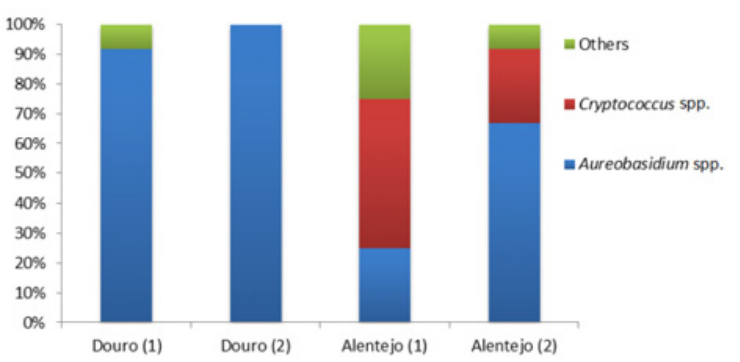

Figure 3. Yeasts isolated from grapes sampled in Douro and Alentejo. Douro (1) - Douro one month before harvesting; Douro (2) - Douro at harvesting; Alentejo (1) - Alentejo one month before harvesting; Alentejo (2) - Alentejo at harvesting.

histamine [8,9]. Yeasts and filamentous fungi isolates were identified by morphological, biochemical and molecular (Internal transcribed spacer gene-ITS) methods [10].

\section{Results and discussion}

A semi-quantitative analysis allowed concluding that bacterial number was higher in Alentejo isolations comparatively to the Douro ones. However, a total number of fungi was approximately the same in both regions at harvesting but one month before was greater in Douro than in Alentejo. Furthermore, and under the same experimental conditions, a much higher number of bacterial morphotypes that correspond to a higher diversity was retrieved in Alentejo (77\%) comparatively to Douro (23\%). The most abundant bacterial phylum obtained was Firmicutes followed by Actinobacteria and Proteobacteria

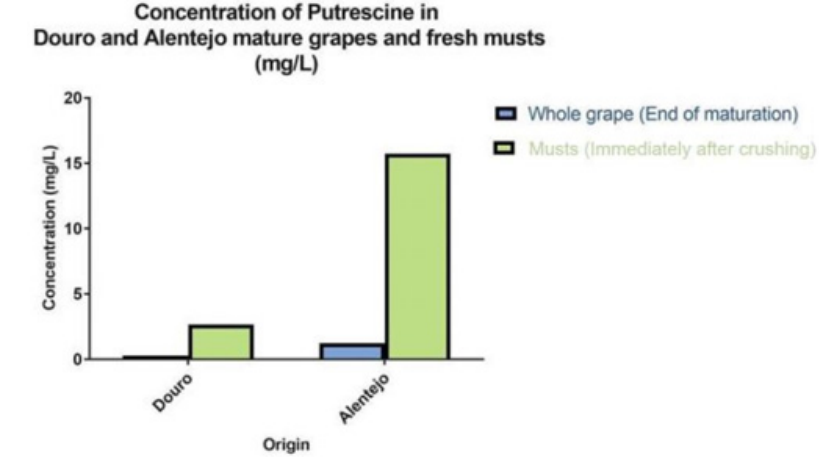

Figure 4. Concentration $(\mathrm{mg} / \mathrm{L})$ of putrescine and tyramine in grapes and musts of Alentejo and Douro, quantified through LC-MS technique.

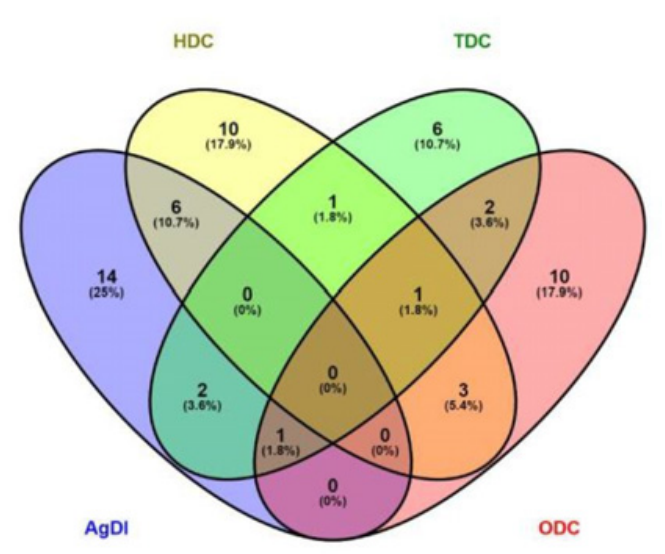

Figure 5. Number of isolates that have the potential to produce one or more BA enzymes (ODC - ornithine decarboxylase, AgDI - agmatine deaminase, HDC - histidine decarboxylase and TDC - tyrosine decarboxylase), by screening of the correspondent gene. $52 \%$ of the studied isolates amplified only the agdi gene, $17.9 \%$ only the $o d c$ gene and the same percentage the $h d c$ gene while only $6(10.7 \%)$ amplified the tyrd gene. Regarding isolates that amplified three genes only $1.8 \%$ amplified for $o d c, h d c$ and tyrd and $1.8 \%$ for the agdi, tyrd and odc. Amplification of agdi and $o d c$ was achieved by $3.6 \%$ of isolates, $1.8 \%$ for the $h d c$ and tyrd, $10.7 \%$ for agdi and $h d c, 5.4 \%$ for $o d c$ and $h d c$ and $3.6 \%$ for $o d c$ and tyrd. None of the isolates amplified the four genes

(Alpha and Gamma classes) but the highest diversity (9 genera) was obtained in the Actinobacteria (Fig. 1).

Considering grape fungal communities, filamentous fungi were predominant when compared to yeasts.

Fungal species of the genus Cladosporium were shown to be prevalent followed by Aspergillus, Penicillium and Alternaria (Fig. 2). Cryptococcus spp. were only found in Alentejo while Aureobasidium was found both in Douro, where it dominated, and in Alentejo where the number increased with maturation (Fig. 3).

Chemical analysis showed that the levels of putrescine detected in mature grapes were higher in Alentejo compared to Douro. A rapid and relevant increase of the levels of putrescine (more than 10 times) was observed especially in Alentejo from mature grapes to fresh musts.

As relevant differences existed between the bacterial grapes communities of Alentejo and Douro, contrarily to what was observed in the fungal communities, bacteria 
were screened for potential BAs-production aiming to establish a correlation between bacteria and the high putrescine levels in musts from Alentejo.

The isolated bacteria revealed a great potential for BAproduction (57\% of the isolates) especially for putrescine (56\% of the isolates) and lower levels of amplifications were obtained for histamine, tyramine and cadaverine (Fig. 5). Isolates with gene amplicons for tyramineproduction were only detected in Douro region.

Due to the (1) high bacterial number, (2) high bacterial diversity and (3) great potential for putrescine production observed in Alentejo grape bacterial community, our results suggest a bacterial grape origin for the high putrescine levels found in fresh musts of this region.

\section{References}

[1] E. Kecskeméti, B. Berkelmann-Löhnertz, A. Reineke, PLoS ONE 11, e0160852 (2016)

[2] V. Loureiro, M. M. Ferreira, S. Monteiro, R. B. Ferreira, In H. Gerós, M. Chaves, S. Delrot (eds) The Biochemistry of the Grape Berry, pp. 241-268 (2012)
[3] N. A. Bokulich, T. S. Collins, C, G. Allen, H. Heymann, S. E. Ebeler, D. A. Mills, mBio 7(3), e00631-16 (2016)

[4] A. Y. Smit, W. J. du Toit, M. du Toit, South Africa Journal of Enology and Viticulture 29, 109-126 (2008)

[5] EFSA, European Food Safety Authority (EFSA) The EFSA Journal 9, 2393. [93 pp.] (2011)

[6] N. Gouveia, Desenvolvimento de uma metodologia analítica para determinação de aminas biogénicas em tunídeos. Master thesis. Funchal: Universidade da Madeira (2009)

[7] A. P. Graça, F. Viana, J. Bondoso, M. I. Correia, L. A. Gomes, M. Humanes, A. Reis, J. Xavier, H. Gaspar, O. M. Lage, Front. Microbiol. 6, 389 (2015)

[8] M. Coton, A. Romano, G. Spano, K. Ziegler, C. Vetrana, C. Desmarais, A. Lonvaud-Funel, P. Lucas, E. Coton, Food Microbiology 27, 1078-1085 (2010)

[9] J. M. Landete, S. Ferrer, I. Pardo, Food Control 18, 1569-1574 (2007)

[10] T. J. White, T. Bruns, S. Lee, J. Taylor. PCR Protocols: a Guide to Methods and Applications 38, 315-22 (1990) 